\title{
EDITORIAL
}

\section{Indiscriminate use of antimicrobials during Covid-19 pandemic}

Antimicrobials have enabled medical advancements over several decades. However, the continuous emergence of resistance to antimicrobials restricts our ability to treat diseases and limits our efforts to achieve health related sustainable development goal. The slogan used for the last five years was "Antibiotics: Handle with Care" has been changed to "Antimicrobials: Handle with Care" in 2020. Antimicrobials includes antibiotics, antivirals, antifungals and antiparasitics - used to prevent and treat infections in humans, animals and plants. ${ }^{1}$

Antimicrobial resistance (AMR) is a neglected global crisis that requires urgent attention and action. Dr Ghebreyesus, (Director General, WHO) warned that the world is losing its ability to use critically important antimicrobial medicines, the pandemic is making it worse.$^{1}$ The Covid-19 pandemic has led to an increased use of antibiotics, which ultimately will lead to higher bacterial resistance rates that will impact the burden of disease and deaths during the pandemic and beyond.

The global threat of antimicrobial resistant bacteria is worsening as many patients admitted to hospital with covid-19 receive antibiotics to keep secondary bacterial infections in check. A review of data from covid-19 cases, mostly in Asia, found that more than $70 \%$ of patients received antimicrobial treatment despite less than $10 \%$, on average, having bacterial or fungal coinfections. ${ }^{2}$ The same study also found frequent use of broad spectrum antibiotics, designed to kill a wide range of bacteria, that can accentuate AMR.

Dr Soldani (Infectious disease specialist) confirmed that, the hospitals in Italy had to deal with increased levels of antibiotic resistant bacteria during the pandemic- such as pseudomonas and enterococci particularly due to widespread use of third generation cephalosporins. Research conducted in nine countries of the European Region showed increasing antibiotic use throughout the pandemic along with cases. Among those taking antibiotics, $79-96 \%$ reported not having been infected with COVID-19 but were taking antibiotics, believing that would prevent infection. ${ }^{2}$ Hospitals, particularly intensive care units, are hotbeds of antimicrobial resistance.

There is a lot of uncertainty about the disease process and the pathology of the infection. When the clinician doesn't have all the necessary information to understand truly what's happening in the patient, it tends to drive more antibiotic use. Antibiotics do not directly affect the respiratory virus responsible for COVID-19, but viral respiratory infections often lead to bacterial pneumonia. Evidence indicates that only up to $15 \%$ of severely affected COVID-19 patients develop bacterial co-infection and may need antibiotics. ${ }^{3}$ Diagnosing coinfections is complex even in the best medical facilities. Although most serum biomarkers lack specificity for differentiating bacterial from viral infection, increased procalcitonin has been considered as a relatively better marker.

Some media reports and political leaders amplified the possible use of the antibiotic azithromycin in combination with the drug hydroxychloroquine, which likely contributed to shortages of both drugs despite the lack of clinical evidence for their effectiveness. Now, the hard lesson from the hydroxychloroquine story for the medical community is that science should not be mixed with politics. A number of articles in the peer reviewed literature over the last several months have consistently demonstrated the lack of efficacy of hydroxychloroquine over COVID-19.4 Moreover, one study showed the addition of azithromycin in combination with antimalarial drugs may induce heart failure and cardiovascular mortality.

Disruptions to health services during the pandemic are causing interruptions to treatments, such as for tuberculosis and human immunodeficiency virus, which can also lead to drug resistance. Similarly, disruption to vaccination services can increase risk of infection, potentially leading to an overuse of antimicrobials. Another potential threat is the wide use of biocidal agents for environmental and personal disinfection. Low level exposure to biocidal agents lead to drug resistant strains and enhance the risk of cross resistance to antibiotics. ${ }^{1}$

The huge shift towards telehealth consultation during the pandemic prompted antibiotic overprescribing. A recent paediatric study demonstrated that overprescribing is much more common in telehealth visits than in-face-to face visits, National Health Service (England) report supports evidence that antibiotic prescribing rates are higher in remote consultations than during in person appointments reflecting greater diagnostic uncertainty from inability to examine patients. However, NICE (National Institute of Health \& Care Excellence) guideline focused on the short course empiric treatment only in suspected cases with high neutrophil count, lobar consolidation and a positive biomarker.

In low and middle income countries (LMIC), high burden of multidrug resistant organisms, prevails due to overemphasis on the role of antimicrobials. ${ }^{2}$ In Bangladesh sanitation, personal hygiene, and social distancing are not adequate. People are generally less concerned about viral or other contagious diseases. Here self medication is very common and people stockpiled antibiotics with the misguided prospect of shielding them against the virus. A recent study found that multidrug resistance (MDR) has rapidly increased in Bangladesh and jumped nearly two fold in 2019 compared to 2015. Experts from India (Prof Jagat Ram) raised concern against Antimicrobial resistance and warned that routine prescription of antibiotics before any medical procedure should be avoided. Nigeria Centre for Disease Control, use their digital platforms to inform the public on 
ineffectiveness of antimicrobials in the treatment for covid -19 .

AMR is one of the top 10 global public health threats facing humanity. Bacterial infections unsuccessfully treated due to AMR claim at least 700,000 lives per year worldwide. ${ }^{3}$ In 2019 , the UN interagency Coordination Group on Antimicrobial Resistance warned that by 2050, AMR diseases could cause 10 million deaths each year.

In recent years, along with surge of AMR there is also decline in newer antibiotic development. The pipeline of new antimicrobials is practically empty. In 2019 WHO identified 32 antibiotics in clinical development that address the WHO list of priority pathogens, of which only six were classified as potentially promising. Since the discovery of Penicillin, in 1928 by Alexander Fleming there has been cognizance of resistance alongside the discovery of new antibiotics. However, these new antibiotics, will suffer the same fate as the current ones, if we do not change the way of use.

For common bacterial infections including urinary tract infections, sepsis and diarrhoea, high rates of resistance against antibiotics have been observed world wide. According to Global Antimicrobial Resistance and Use Surveillance System (GLASS), the rate of resistance to ciprofloxacin, varied from 8 to $92 \%$ for Escherichia coli and from $4 \%$ to $79 \%$ for Klebsiella pneumoniae. In some countries, carbapenem antibiotics do not work in more than $50 \%$ of patients treated for $\mathrm{K}$. pneumoniae infections. Bacteria, resistant to colistin, the last resort treatment have also been detected in several countries. People with methicillin-resistant Staphylococcus aureus (MRSA) infections are $64 \%$ more likely to die than people with drug sensitive infections. Each year in the U.S., at least 2.8 million people are infected with antibiotic resistant bacteria or fungi and more than 35,000 people die as a result. ${ }^{5}$ In UK, Antimicrobial resistant blood stream infections have increased by 32\% since 2015 .

The southeast Asia is considered to have the highest risk of AMR among all the WHO regions. Here the contributory factors are- wide availability of over the counter (OTC) drug, presence of unqualified providers in the informal sector, unethical aggressive marketing practices of the pharmaceutical companies, nonhuman antibiotic use and the weak surveillance system. High level of infections in LMICs are attributed to lack of access to clean water, sanitation and hygiene (WASH), poor prevention and control of infection and disease in health care facilities, and poor access to quality medicines, vaccines and diagnostics.

In an extensive review article, Researcher from Bangladesh showed that $\mathrm{E}$ coli resistance pattern to antibiotics were significantly high- Amoxyclav (68\%), Ciprofloxacin (65\%), Cefotaxime (55\%), Ceftriaxone (59\%) and for Klebsiella it was 58\%, 77\%, 99\%, 78\% respectively. Although the study area is limited to
Bangladesh, resistant species can spread throughout the world by means of mankind, animals, water and air. ${ }^{5}$

AMR is a complex problem that requires a united multisectoral approach. The One Health approach brings together multiple sectors and stakeholders engaged in human terrestrial and aquatic animal, plant health and food production. Globally, countries associated with Global Action Plan (GAP) on AMR during the 2015 World Health Assembly are committed to the development and implementation of multisectoral national action plans. ${ }^{1}$ World Antimicrobial Awareness Weak (WAAW-18 to 24 November), held annually since 2015 is a global campaign that aims, to raise awareness of antimicrobial resistance worldwide and encourage best practices among the general public, health workers and policy makers.

Isabel Oliver (Public Health Director, England) stressed on the role of public in tackling antimicrobial resistance. Taking antibiotics without need can have grave consequences for us and our family's health now and in the future. Last year, the WHO urged Governments to implement the AWaRe tool, which classifies antibiotics into three groups- access, watch, and reserve to enable clinicians to know which antibiotics to use for common infections, which ones should be used with caution, and which should be used only as a last resort. ${ }^{2}$

The WHO guidance to medical persons urged them not to provide antibiotic treatment to patients with mild Covid-19, and emphasised the need for proper assessment and diagnostic testing before antibiotic use in moderate and severe cases. Given the existing widespread AMR and the knowledge gained in the current management, Health workers can prevent another superbug pandemic by restricting the indiscriminate use of antimicrobials.

CH Rasul, Professor of Paediatrics, KCMC\&H (Email: chrasul@yahoo.com)

\section{References}

1. WHO. Preventing the COVID-19 pandemic from causing an antibiotic resistance catastrophe. WHO Bulletin 18 Nov 2020

2. Hsu J. How covid-19 is accelerating the threat of antimicrobial resistance - Healthcare responses to the novel coronavirus may be hastening another long looming public health threat BMJ 2020; 369: m1983

3. Armitage R, Nellums LB. Antibiotic prescribing in general practice during COVID-19. Lancet Infect Dis 2020; Dec 1: S1473-3099

4. Rosenberg ES, Dufort EM, Udo T, et al. Association of treatment with hydroxychloroquine or azithromycin with in hospital mortality in patients with COVID-19 in New York state. JAMA 2020; 323: 2493-2502

5. Rawson TM, Moore LSP, Zhu N, et al. Bacterial and fungal co infection in individuals with coronavirus: A rapid review to support COVID -19 antimicrobial prescribing. Clin Infect Dis 2020; $71: 2459-68$ 